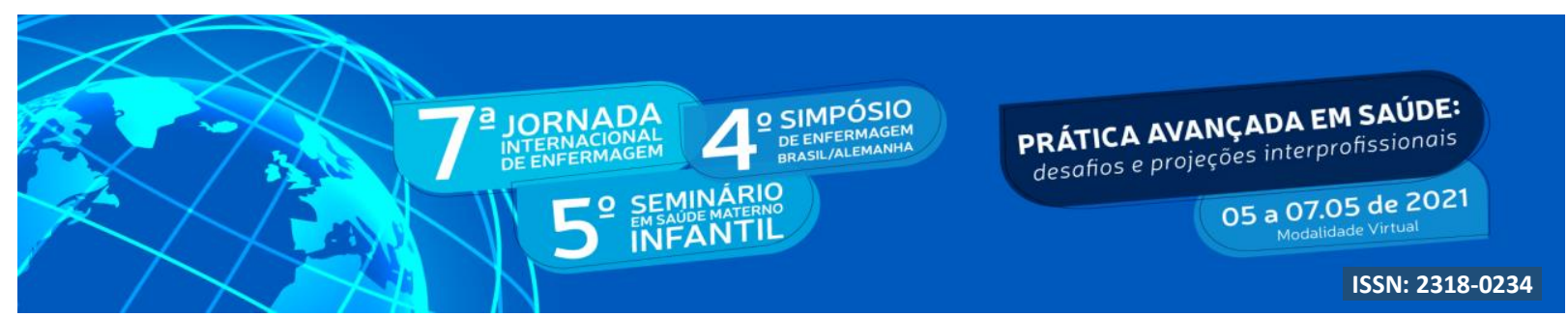

DOI: http://doi.org/10.48195/jie2021-106

\title{
CONSULTA DE ENFERMAGEM NO PRÉ-NATAL DE RISCO HABITUAL: IMPLEMENTAÇÃO EM UMA UNIDADE BÁSICA ${ }^{1}$
}

\author{
Mariana Braga da Silva²; Ângela Maria Naidon³; Rosiane Filipin Rangel ${ }^{4}$
}

\begin{abstract}
RESUMO
Objetivou-se relatar a experiência de enfermeiras em uma Unidade Básica de Saúde na implementação da consulta de enfermagem no pré-natal de risco habitual. Metodologia: Trata-se de um relato de experiência realizado por enfermeiras em uma Unidade Básica de Saúde de um município na região do Vale do Rio Pardo, no período de março de 2019 até fevereiro de 2020. Resultados e Discussão: A consulta de pré-natal de enfermagem iniciou-se, gradualmente, de maneira que foi sendo implementada na rotina das enfermeiras e das equipes. Conclusão: Considera-se que a prática de pré-natal das enfermeiras está em processo de consolidação no município. E, é uma maneira de assegurar atendimento integral as gestantes e oferecer um olhar mais amplo no que se refere ao acolhimento as demandas que existem em um contexto gestacional junto também à família.
\end{abstract}

Palavras-chave: Cuidado Pré-natal; Enfermagem; Atenção Primária à Saúde.

\begin{abstract}
The objective was to report the experience of nurses in a Basic Health Unit in the implementation of nursing consultation in prenatal care at usual risk. Methodology:This is an experience report carried out by nurses in a Basic Health Unit in a municipality in the Vale do Rio Pardo region, from March 2019 to February 2020. Results and discussion:The prenatal nursing consultation begins, gradually, in a way that was being implemented in the routine of nurses and teams. Conclusion: It is considered that the prenatal practice of nurses is in the process of consolidation in the municipality. And it is a way of ensuring comprehensive care as pregnant women and offering a broader look with regard to embracement as demands that exist in a gestational context with the family as well
\end{abstract}

Key Words: Prenatal Care; Nursing; Primary Health Care

\footnotetext{
${ }^{1}$ Trabalho de pesquisa.

${ }^{2}$ Enfermeira e Especialista em Saúde Materna e Neonatal pela Universidade Franciscana (UFN). Hemocentro Regional de Pelotas - Pelotas, RS. E-mail: maiabragasilva@gmail.com

${ }^{3}$ Enfermeira. Mestre em Enfermagem pela Universidade Federal de Santa Maria (UFSM). Especialista em Enfermagem Obstétrica pela Universidade Franciscana (UFN). Hospital Casa de Saúde - Santa Maria, RS. Email: angelanaidom@yahoo.com.br

${ }^{4}$ Orientadora. Enfermeira. Doutora em Enfermagem pela Universidade Federal do Rio Grande (FURG). Docente do curso de Enfermagem e do Mestrado Profissional em Saúde Materno Infantil da Universidade Franciscana (UFN) - Santa Maria, RS. E-mail: rosiane@ufn.edu.br
} 


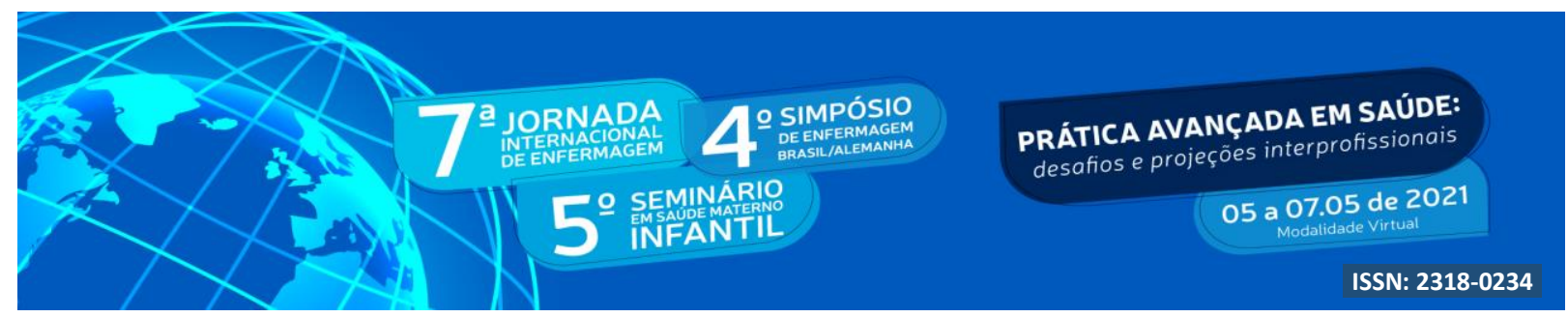

\section{INTRODUÇÃO}

Nos sistemas de saúde, tem-se priorizado o cuidado da mulher no campo da saúde reprodutiva, com foco na atenção ao pré-natal, parto, puerpério e planejamento reprodutivo, orientação de grande relevância social e epidemiológica. No entanto, o atendimento integral das mulheres - com acolhimento de suas demandas e necessidades, garantia do acesso e respostas a contento - ainda está em processo de consolidação (BRASIL, 2016).

Embora, nas últimas décadas, a cobertura de atenção ao pré-natal tenha aumentado, garantir sua qualidade permanece como o maior desafio. Essa melhoria refere-se a uma mudança sensível na atitude dos profissionais de saúde e na eficiência e presteza dos serviços (CALIFE; LAGO; LAVRAS, 2010).

O objetivo do acompanhamento pré-natal é assegurar o desenvolvimento da gestação, permitindo o parto de um recém-nascido saudável, sem impacto para a saúde materna, inclusive abordando aspectos psicossociais e as atividades educativas e preventivas (BRASIL, 2012).

O acompanhamento da gestante e da puérpera na Atenção Básica deve ser multiprofissional, com ênfase na participação do profissional médico e de enfermagem. $\mathrm{O}$ pré-natal classificado como baixo risco pode ser realizado pelo enfermeiro, de acordo com o Ministério da Saúde e conforme garantido pelo Decreto $n^{\circ} 94.406 / 87$, que regulamenta a Lei de Exercício da Enfermagem (COFEN, 1987).

A atuação do enfermeiro no pré-natal tem assumido papel de destaque como preconizado no programa de assistência ao pré-natal de baixo risco do Ministério da Saúde. Segundo o protocolo ministerial cabe ao enfermeiro, entre outras atribuições: realizar consulta de enfermagem, solicitar exames de rotina, realizar anamnese e exame físico, fazer busca ativa das gestantes faltosas, realizar captação precoce até 120 dias de gestação, avaliar cartão de vacinas e desenvolver um papel importante na área educativa e de humanização (RODRIGUES; NASCIMENTO; ARAÚJO, 2011).

Também, o enfermeiro durante as consultas de pré-natal possui a autonomia de realizar as testagens rápidas para Infecções Sexualmente Transmissíveis (IST) como - Vírus da Imunodeficiência Humana (HIV), Sífilis, Hepatite B e Hepatite C - tanto na gestante quanto no seu companheiro, pré-natal do parceiro. 


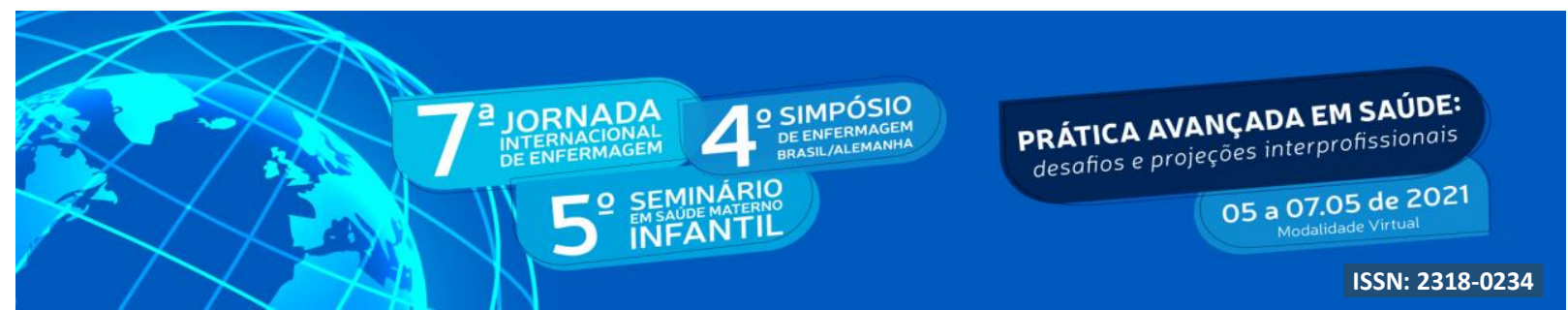

Nesse sentido, cabe ressaltar que, a Política Nacional de Atenção Integral à Saúde do Homem (PNAISH) instituída pela Portaria GM/MS n 1.944, de 27 de agosto de 2009, aposta na perspectiva da inclusão do tema da paternidade e cuidado, por meio do pré-natal do parceiro, nos debates e nas ações voltadas para o planejamento reprodutivo como uma estratégia essencial para qualificar a atenção à gestação, ao parto e ao nascimento, estreitando a relação entre trabalhadores de saúde, comunidade e, sobretudo, aprimorando os vínculos afetivos familiares dos usuários e das usuárias nos serviços ofertados (HERRMANN et al., 2016).

Durante a consulta de enfermagem, além da competência técnica, o enfermeiro deve demonstrar interesse pela gestante e pelo seu modo de vida, ouvindo suas queixas e considerando suas preocupações e angústias. Para isso, o enfermeiro deve fazer uso de uma escuta qualificada, a fim de proporcionar a criação de vínculo. Assim, ele poderá contribuir para a produção de mudanças concretas e saudáveis nas atitudes da gestante, de sua família e comunidade, exercendo um papel educativo (BRASIL, 2012).

O período pré-natal é uma época de preparação física e psicológica para o parto e para a maternidade e, como tal, é um momento de intenso aprendizado e uma oportunidade para os profissionais da equipe de saúde desenvolverem a educação como dimensão do processo de cuidar (RIOS;VIEIRA, 2004).

\section{OBJETIVO}

Relatar a experiência de enfermeiras em uma Unidade Básica de Saúde na implementação da consulta de enfermagem no pré-natal de risco habitual

\section{METODOLOGIA}

Trata-se de um relato de experiência acerca da implementação da consulta de enfermagem no pré-natal de baixo risco realizado por enfermeiras em uma Unidade Básica de Saúde. A experiência é como um objeto de análise para o relato de experiência, uma fonte inesgotável de sentidos e possibilidades passíveis de análises. O relato de experiência está compreendido como um trabalho de linguagem, uma construção que não objetiva propor a última palavra, 


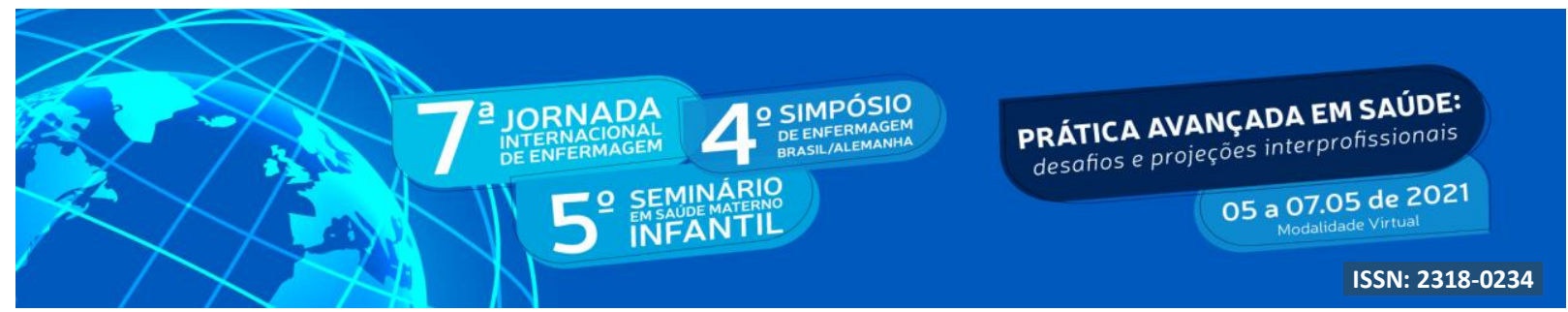

mas que tem caráter de síntese provisória, aberta à análise e a permanente produção de saberes novos e transversais (DALTRO; FARIA, 2019).

O pré-natal de risco habitual está sendo implementado de forma gradual e constante, no município descrito a seguir, baseado nos moldes e protocolos preconizados pelo Ministério da Saúde. Deste modo, o início da implementação das consultas de enfermagem com as gestantes e seus parceiros realizou-se de março de 2019 até fevereiro de 2020.

A referida unidade de saúde localiza-se na região do Vale do Rio Pardo no Rio Grande do Sul, a 145,9 km da capital. Com população estimada de 38.275 pessoas, possui área territorial de 2.050,650 km², entre área rural e área urbana (IBGE, 2010). No âmbito da saúde, abrange 84\% de equipes de atenção básica (SECRETARIA ESTADUAL DE SAÚDE, 2019).

A Unidade Básica de Saúde (UBS) Central é considerada a principal referência e porta de entrada da atenção primária em saúde do município. Abrange todas as áreas nos quais não estão vinculadas as Estratégias de Saúde da Família (ESF) e mais toda a extensa área rural. A UBS Central está dividida por setores como: recepção, sala de triagem, regulação, vigilância epidemiológica - setor de vacinação e programa de IST/Aids, pediatria, grupo de hipertensos e diabéticos - Hiper/Dia, ambulatório de pequenos procedimentos - como curativos e pequenas cirurgias, consultório odontológico, salas de consultas médicas, atendimento de nutrição, Estratégia de Agentes Comunitários de Saúde (EACS) - no qual abrange todas os Agentes Comunitários de Saúde (ACS) que não são vinculados a uma ESF do município - e o setor de saúde da mulher.

Dentro desse cenário, a UBS Central apresenta uma diversidade de equipes, as quais, na grande maioria, possuem coordenação de enfermagem, com 8 enfermeiras para realizar a organização dos processos de trabalho. No total, são em torno de 53 colaboradores na unidade e mais 32 ACS que trabalham em suas microáreas com coordenação direta dentro da UBS Central. Tem-se, em média, um fluxo de atendimentos ao mês de 123 mulheres entre gestantes e puérperas, dentro de todo o período gestacional e pós-parto.

\section{RESULTADOS E DISCUSSÃO}

Em Fevereiro de 2019 realizou-se uma reunião com o Secretário Municipal de Saúde e avaliou-se a necessidade de dar início às consultas de pré-natal da enfermagem para gestantes 


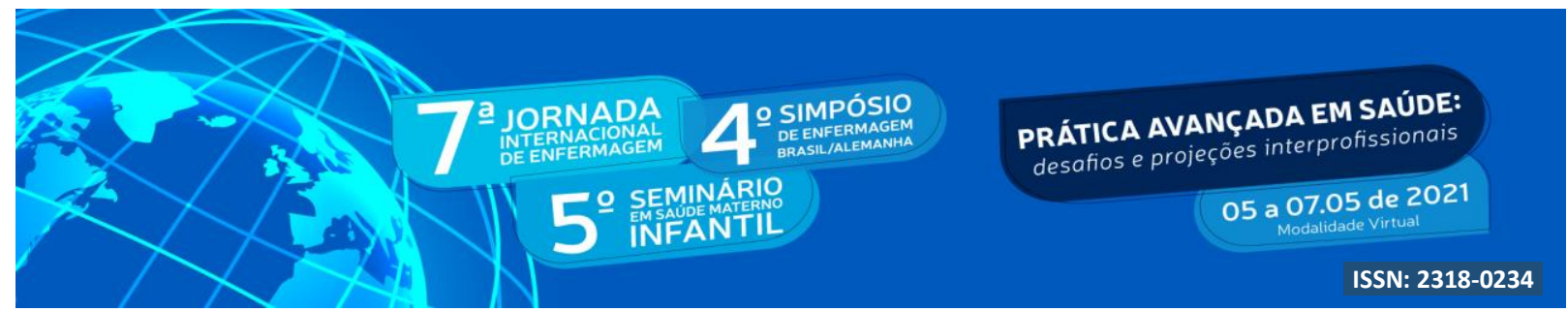

de risco habitual dentro da UBS Central, já que anteriormente tais consultas eram centralizadas na figura do médico ginecologista/obstetra. Sendo assim, Cardoso et al. (2016) considera que o pré-natal com qualidade é uma das medidas a serem buscadas, principalmente pelos gestores de saúde, quando se pretende combater as taxas de morbimortalidade materna e perinatal. A consulta de enfermagem é uma atividade independente, realizada privativamente pelo enfermeiro, e tem como objetivo proporcionar condições para a promoção da saúde da gestante e a melhoria na sua qualidade de vida, mediante uma abordagem contextualizada e participativa (BRASIL, 2012).

Para tanto, organizou-se uma agenda de pré-natal da enfermagem, a partir de uma conversa com três enfermeiras do serviço responsáveis pelas gestantes e seu contexto biopsicossocial, com o intuito de iniciar as marcações das consultas conforme as mulheres, em idade fértil, aparecessem com o exame de Beta-HCG (Gonadotrofina Coriônica Humana) positivo/reagente. Sendo assim a proposta foi iniciada em março de 2019.

Para melhor contextualizar o serviço, as enfermeiras da UBS Central que tem maior envolvimento com gestantes são de dois setores diferentes: uma enfermeira é responsável pelo setor de saúde da mulher, no qual abrange o atendimento da maioria das gestantes do município, tanto as de risco habitual como também as de alto risco, já que a UBS é referência municipal nesse atendimento, pois é o local onde se tem atendimento médico ginecologista/obstetra. Já as outras duas enfermeiras são do setor EACS que coordenam diretamente as ACS das áreas de distritos rurais e mais três microáreas de abrangência urbana que não possuem ESF.

Dessa maneira, iniciaram-se as consultas de pré-natal de enfermagem com o objetivo de criar maior vínculo destas gestantes com o serviço e acolhimento para suas demandas, uma vez que durante o ciclo gestacional a mulher necessita de apoio e segurança durante o seu prénatal. Práticas acolhedoras proporcionam a identificação das principais vulnerabilidades das gestantes levando em consideração seu contexto social, assim como a criação de um vínculo de confiança entre o profissional e a gestante, permitindo uma maior aceitação e continuidade dessas mulheres no serviço (BRASIL, 2002).

A prática de assistência ao pré-natal na UBS Central era, totalmente, centralizada em quatro médicos(as) ginecologistas/obstetras. Porém, com a implementação do atendimento de 


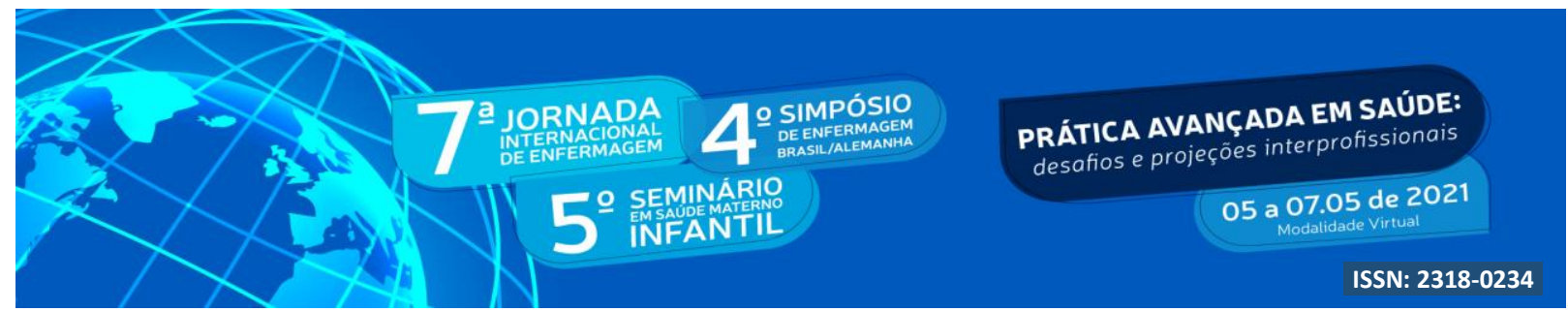

enfermagem, houve o primeiro impacto de como seria essa assistência e sua aceitação pelas mulheres em período gravídico.

Inicialmente, o agendamento da primeira consulta de pré-natal é realizado a partir do resultado de exame laboratorial que confirma a gravidez, e após um acolhimento para ver qual a localidade esta gestante pertence. A partir dessa informação direciona-se para qual enfermeira vai ser agendada a consulta. Também, muitas vezes, com um papel muito importante na Atenção Básica, o ACS é quem traz a demanda de necessidade do agendamento de novas gestantes das microáreas que atuam. Para o Ministério da Saúde, um dos papéis do ACS é auxiliar na captação e orientação para que toda gestante inicie o mais precocemente o acompanhamento pré-natal (BRASIL, 2009).

Os dias da semana para consulta de pré-natal de enfermagem são de acordo com a disponibilidade dos consultórios, principalmente nos turnos da tarde. Também, observou-se a necessidade de ter horário específico para cada gestante e seu parceiro virem na consulta (prénatal do parceiro), para que não se tenha espera dos mesmos. Por esse motivo, são agendadas até duas consultas no período, já que cada momento leva em torno de uma hora ou até uma hora e trinta minutos.

A consulta de pré-natal de enfermagem iniciou-se, gradualmente, de maneira que foi sendo implementada na rotina das enfermeiras e das equipes, tornando-se uma nova estratégia da Atenção Básica no município para prestar um cuidado integral como preconizado pelo Ministério da Saúde e pela Secretaria Estadual de Saúde do Rio Grande do Sul.

Após o processo de implementação ter iniciado, observou-se insegurança por parte das enfermeiras, no que diz respeito ao novo e ao que poderiam encontrar na singularidade de cada gestante, principalmente, para as duas enfermeiras do setor EACS, as quais não tinham como rotina do trabalho acompanhamento de consultas de pré-natal. Contudo, os protocolos assistenciais na Atenção Primária para a gestação do Ministério da Saúde foram de grande relevância e conhecimento para prestar o cuidado.

No entanto, a questão de protocolos municipais ainda é o maior impasse que a enfermagem enfrenta, já que não se têm regulamentado no município aqueles que liberam a solicitação, por parte do profissional enfermeiro, de exames e prescrição de medicamentos, como prevê o Decreto No 94.406/87 da Regulamentação do Exercício da Enfermagem, que no Art. $8^{\circ}$ incumbe ao enfermeiro como integrante da equipe de saúde: prescrição de 


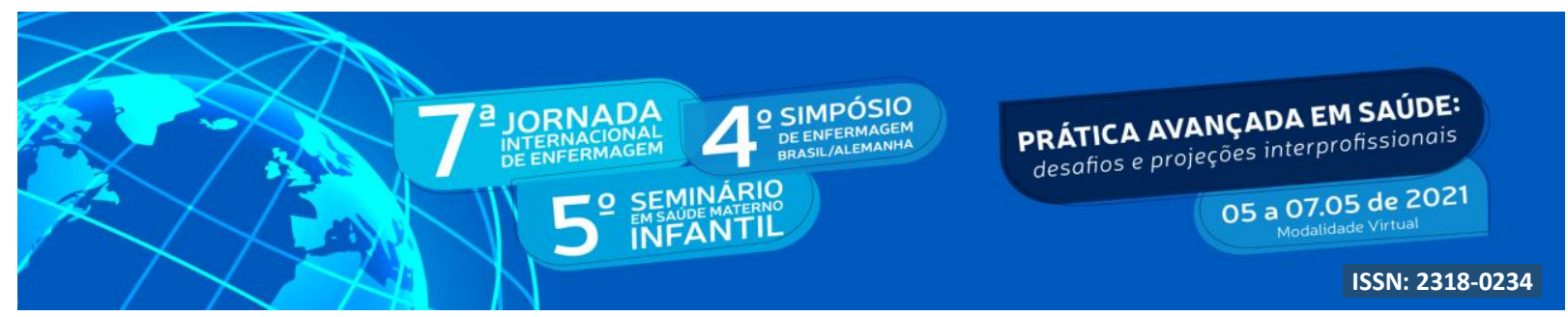

medicamentos previamente estabelecidos em programas de saúde pública e em rotina aprovada pela instituição de saúde (COFEN, 1987).

Os exames do pré-natal podem ser solicitados por profissionais da medicina e enfermagem que realizam o acompanhamento do pré-natal de gestantes e seu parceiro, conforme protocolos assistenciais (SORTICA et al., 2018). Assim, até o momento deste relato, a primeira consulta de pré-natal no município é um trabalho em conjunto entre enfermeiras e médicos para solicitar os primeiros exames laboratoriais, de imagem e prescrição de suplementação de ácido fólico e sulfato ferroso, conforme preconizado pelo Ministério da Saúde.

Durante esse período, de experiências vivenciadas, pode-se analisar a aceitação das gestantes e seus parceiros. Observou-se, desde o primeiro acolhimento e contato, já no momento do agendamento da consulta de enfermagem, um estranhamento por parte da maioria das mulheres - preocupadas se iriam consultar com o médico/obstetra em algum momento da gestação. Para as mesmas, é explicado que as consultas serão alternadas entre enfermagem e medicina, e fica evidente a surpresa das gestantes com a novidade.

No início da implementação, nos primeiros dois meses, houve algumas gestantes que não compareceram na consulta agendada, então era realizado busca ativa das mesmas, principalmente pelas ACS, e realizado novo agendamento. Dessas gestantes faltosas, algumas afirmaram, não terem ido à consulta, por acharem que não era importante estar agendado com a enfermeira. Porém, aproveitou-se a oportunidade das consultas de pré-natal com a enfermeira para esclarecer dúvidas de como será o novo acompanhamento e demonstrar segurança e acolhimento das prioridades para aquela gestante e companheiro. E esses, ao final de cada consulta, mostravam-se satisfeitos e tranquilos.

Ressalta-se, ainda, que durante o mesmo período de implementação da consulta de enfermagem no pré-natal, houve as primeiras reuniões da Rede Cegonha no município, entre profissionais da Atenção Básica e hospitalar, que vieram a fortalecer a rede de cuidados à mulher gestante. Através da PORTARIA No 1.459, DE 24 DE JUNHO DE 2011 a Rede Cegonha, instituída no âmbito do Sistema Único de Saúde (SUS), consiste numa rede de cuidados que visa assegurar à mulher o direito ao planejamento reprodutivo e à atenção humanizada à gravidez, ao parto e ao puerpério, bem como à criança o direito ao nascimento seguro e ao crescimento e ao desenvolvimento saudáveis (BRASIL, 2011). 




\section{CONCLUSÃO}

A implementação da consulta de enfermagem no pré-natal é uma maneira de assegurar atendimento integral as mulheres gestantes e oferecer um olhar mais amplo no que se refere ao acolhimento as demandas que existem em um contexto gestacional junto também à família. A enfermagem se mostra muito importante nesses atendimentos, já que são profissionais, que dentro de suas atribuições, apresentam autonomia para acompanhar gestantes de risco habitual e característica de humanização, com olhar ampliado para as necessidades que envolvem toda a gestação.

Sabe-se que o enfermeiro tem uma das atuações principais nos serviços de saúde, porém ainda existe um receio por parte da população atendida, principalmente as gestantes, que antes só viam o profissional médico como referência, e a partir dessa iniciativa de implementação do pré-natal de enfermagem, já estão tendo as enfermeiras como maior apoio dentro da UBS Central para questionar dúvidas, e dividir inseguranças que permeiam a gestação.

Ainda que, a realização da prática de pré-natal das enfermeiras esteja em processo de consolidação, o objetivo maior será estabelecer este processo de consultas de enfermagem para as demais unidades de saúde do município. Também, como consequência deste relato, a criação do Protocolo de Saúde da Mulher Municipal é uma das próximas metas a serem colocadas em prática, para melhorar e respaldar os cuidados e avaliações da enfermagem, tanto para gestantes quanto seus parceiros.

Para que a implementação do pré-natal de risco habitual com a enfermagem continue se fortalecendo dentro do município, torna-se importante investir em educação permanente e continuada das profissionais enfermeiras. Oportunidades, como desse relato de experiência, que a gestão da Secretaria de Saúde Municipal proporcionou, em acreditar no início de algo novo para o município, mas que faz toda diferença no cuidado das gestantes e suas famílias.

\section{REFERÊNCIAS}

BRASIL. Ministério da Saúde. Guia prático do Agente Comunitário de Saúde. Ministério da Saúde, Secretaria de Atenção à Saúde, Departamento de Atenção Básica. Brasília: 


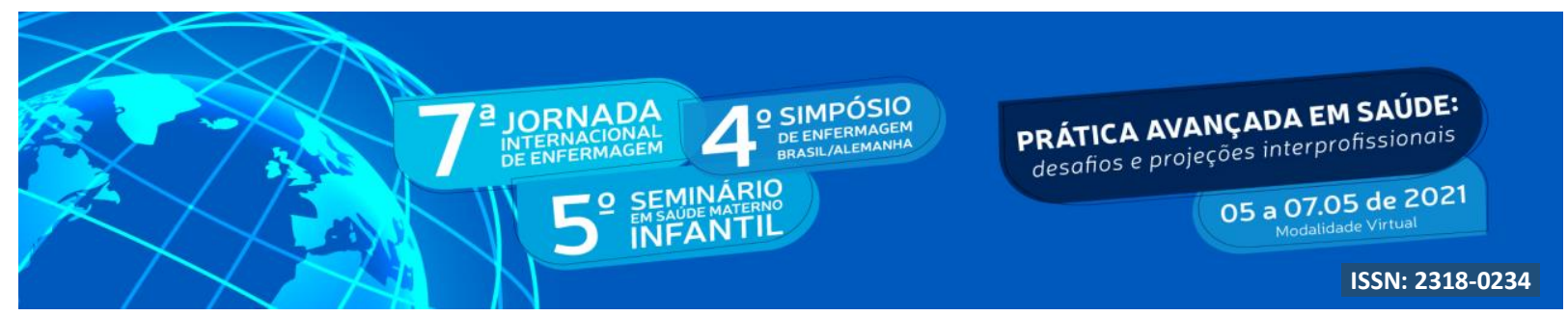

Ministério da Saúde, 2009. Disponível em:

<http://189.28.128.100/dab/docs/publicacoes/geral/guia_acs.pdf >. Acesso em: 04 dec. 2020.

BRASIL. Ministério da Saúde. Política Nacional de Atenção Integral à Saúde do Homem (PNAISH). Instituída pela Portaria GM/MS nº 1.944, de 27 de Agosto de 2009. Disponível em:

<http://bvsms.saude.gov.br/bvs/publicacoes/politica_nacional_atencao_saude_homem.pdf $>$. Acesso em: 04 dec. 2020.

BRASIL. Ministério da Saúde. Protocolos da Atenção Básica: Saúde das Mulheres / Ministério da Saúde, Instituto Sírio-Libanês de Ensino e Pesquisa - Brasília: Ministério da Saúde, 2016. Disponível em:

<http://bvsms.saude.gov.br/bvs/publicacoes/protocolos_atencao_basica_saude_mulheres.pdf $>$ . Acesso em: 04 dec. 2020.

BRASIL. Ministério da Saúde. Rede Cegonha. Instituída pela Portaria n ${ }^{\circ} 1.459$, de 24 DE Junho de 2011. Disponível em:

<http://bvsms.saude.gov.br/bvs/saudelegis/gm/2011/prt1459_24_06_2011.html > . Acesso em 03 dec. 2020.

BRASIL. Ministério da Saúde. Secretaria de Atenção à Saúde. Departamento de Atenção Básica. Atenção ao pré-natal de baixo risco / Ministério da Saúde. Secretaria de Atenção à Saúde. Departamento de Atenção Básica. - Brasília: Editora do Ministério da Saúde, 2012. Disponível em:

〈http://bvsms.saude.gov.br/bvs/publicacoes/cadernos_atencao_basica_32_prenatal.pdf $>$. Acesso em 03 dec. 2020.

BRASIL. Ministério da Saúde. Secretaria Executiva. Humanização do parto. Humanização no pré-natal e nascimento. Brasília (DF), 2002. Disponível em:

<https://bvsms.saude.gov.br/bvs/publicacoes/parto.pdf> . Acesso em 04 dec. 2020.

CALIFE, K; LAGO, T; LAVRAS, C. Atenção à gestante e à puérpera no SUS - SP: manual técnico do pré-natal e puerpério. São Paulo: SES/SP, 2010. Disponível em: $<$ https://www.portaldaenfermagem.com.br/downloads/manual-tecnico-prenatal-puerperiosus.pdf>. Acesso em: 03 dec. 2020.

CARDOSO, M.D; RIBEIRO, C.M.S; OLIVEIRA, I.B; et al. Percepção de gestantes sobre a organização do serviço/assistência em um pré-natal de baixo risco de Recife. Revista de pesquisa: Cuidado é fundamental online; out/dez; v.8, n.4, p.5017-5024, 2016. Disponível em: $<$ http://www.seer.unirio.br/index.php/cuidadofundamental/article/view/4941 $>$. Acesso em: 03 dec. 2020.

COFEN - Lei do Exercício da Enfermagem - Decreto No 94.406/87 da Regulamentação do Exercício da Enfermagem, 1987. Disponível em: $<$

http://www.planalto.gov.br/ccivil_03/decreto/1980- 


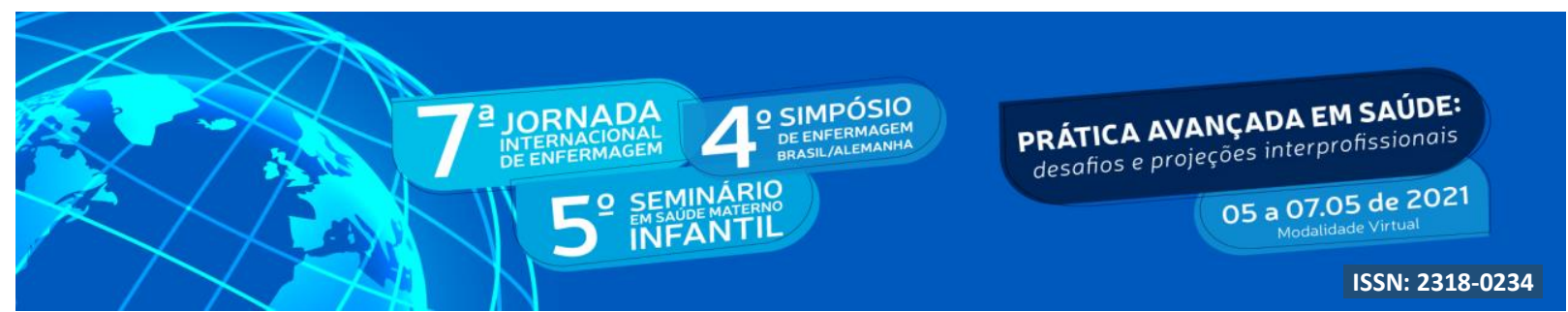

1989/d94406.htm\#: :text=1\%C2\%BA\%200\%20exerc\%C3\%ADcio\%20da\%20atividade,Con selho\%20Regional\%20de\%20Enfermagem\%20da> Acesso em: 03 dec. 2020.

DALTRO, M. R.; FARIA, A. A. Relato de experiência: Uma narrativa científica na pósmodernidade. Estud. pesqui. psicol., Rio de Janeiro, v. 19, n. 1, p. 223-237, jan. 2019. Disponível em http://pepsic.bvsalud.org/scielo.php?script=sci_arttext\&pid=S180842812019000100013\&lng=pt\&nrm=iso . Acesso em: 03 dec. 2020.

HERRMANN, A; SILVA, M.L; CHAKORA, E.S; LIMA, D.C. Guia do Pré-Natal do Parceiro para Profissionais de Saúde. Rio de Janeiro: Ministério da Saúde, 2016. Disponível em: < http://bvsms.saude.gov.br/bvs/publicacoes/guia_pre_natal_parceiro_profissionais_saude.pdf $>$. Acesso em 03 dec. 2020.

IBGE - Instituto Brasileiro de Geografia e Estatística - Indicadores sociodemográficos e de saúde no Brasil. Rio de Janeiro: Instituto Brasileiro de Geografia e Estatística, 2010.

RIOS, C.T.F.; VIEIRA, N.F.C. Ações educativas no pré-natal: reflexão sobre a consulta de enfermagem como um espaço para educação em saúde. Ciênc. saúde coletiva, Rio de Janeiro, v. 12, n. 2, p. 477-486, Apr. 2007 . Disponível em: http://www.scielo.br/scielo.php?script=sci_arttext\&pid=S141381232007000200024\&lng=en\&nrm=iso . Acesso em: 03 Dec. 2020.

RODRIGUES, E; NASCIMENTO, R; ARAÚJO, A. Protocolo na assistência pré-natal: ações, facilidades e dificuldades dos enfermeiros da Estratégia de Saúde da Família. Rev Esc Enferm USP, São Paulo, v.45, n.55, p.1041-7, 2011. Disponível em: $\langle$ https://www.scielo.br/scielo.php?script=sci_arttext\&pid=S0080-62342011000500002>. Acesso em: 04 dec. 2020.

SECRETARIA ESTADUAL DE SAÚDE. Portal BI Gestor Municipal BI, 2019. Disponível em: $<\underline{\text { http://bipublico.saude.rs.gov.br/index.htm>. Acesso em: 27/02/2020 }}$

SORTICA, A.C; CARDOZO G.H; SILVA, G.L; AZEVEDO, M; LEMOS, N.A. Guia do pré-natal da atenção básica - Porto Alegre: Secretaria de Estado da Saúde/ RS, 2018. 